\title{
Les montagnes russes du clonage thérapeutique
}

$>$ Comme chacun le sait depuis un article très médiatisé paru en février de cette année [1], des scientifiques coréens ont obtenu de nombreux embryons humains clonés, et ont même établi une lignée de cellules souches à partir de l'un d'eux. Ce résultat important était assez inattendu compte tenu des difficultés récemment décrites pour le transfert nucléaire chez les primates [2]. À l'évidence, il relance les espoirs et les polémiques sur le clonage thérapeutique - et, au-delà, sur les perspectives de clonage reproductif humain.

\section{L’histoire récente}

Depuis la naissance de Dolly, et après les relatifs succès du clonage animal (une bonne douzaine d'espèces clonées à ce jour, bien que le «rendement» reste faible et que les animaux obtenus présentent souvent des anomalies), l'application de cette technologie à l'espèce humaine a été envisagée [3] ${ }^{1}$. Elle peut prendre deux formes: le clonage reproductif, presque universellement condamné, et ce que l'on a appelé clonage thérapeutique, même si pour certains [4] cette appellation est abusive $(\rightarrow)$.

II s'agirait dans ce cas de constituer un embryon à partir d'un $\rightarrow) \mathrm{m} / \mathrm{s}$ $2002, n^{\circ} 4$, p. 503 ovule énucléé et d'une cellule somatique provenant de la personne dont l'état nécessite une greffe cellulaire. Après développement de l'embryon in vitro jusqu'au stade blastocyte, on prélèverait les cellules souches pour les multiplier au laboratoire puis les pousser à se différencier vers le type cellulaire nécessaire pour traiter le patient par une greffe - a priori bien acceptée puisque génétiquement autologue.

La seule véritable publication dans ce domaine était jusqu'ici celle de l'entreprise Advanced Cell Genetics [5], parue en 2001 dans une revue en ligne d'audience modeste. Les résultats obtenus n'étaient pas merveilleux: le développement des embryons construits par transfert nucléaire n'avait pas été au-delà de six à huit cellules, très loin donc du point permettant d'espérer l'obtention d'une lignée de cellules souches. La même firme avait ensuite annoncé, début 2004, être allée jusqu'au stade de 16 cellules, mais sans que ceci fasse l'objet d'une publication². De son côté, Panos Zavos, l'un des rares scientifiques affirmant tenter un clonage reproductif humain, avait publié en avril 2003 (et dans une autre revue en ligne assez confidentielle) un très bref article présentant un embryon obtenu par transfert nucléaire et

1 Pour une mise au point récente sur l'ensemble du domaine, voir [3].

${ }^{2}$ Ces résultats étaient seulement rapportés dans le magazine grand public Wired.

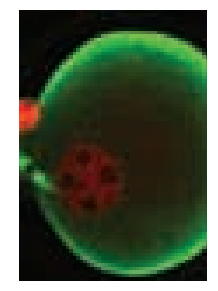

qui s'était développé jusqu'à comporter 8 à 10 cellules [6]. Début 2004, il annonçait

l'implantation d'un tel embryon chez une femme, puis, deux semaines plus tard, l'échec de cet essai : la grossesse n'avait pas «pris». Indépendamment des objections de principe au clonage reproductif humain, soulignons le caractère irresponsable d'une telle tentative, compte tenu du risque très élevé d'anomalies embryonnaires et de l'impossibilité de s'assurer réellement du bon état de l'embryon avant implantation. Elle a au moins le mérite de nous rappeler une fois encore que clonage reproductif et clonage thérapeutique débutent exactement de la même manière - ce qui est une des raisons de l'opposition au second, car sa mise au point favoriserait le passage au premier...

\section{Des primates problématiques}

La relative modestie de ces succès pouvait faire penser que le clonage humain restait une perspective lointaine. Cela d'autant plus que, fin 2003, des études effectuées sur le singe Rhésus avaient identifié une difficulté propre aux primates [2]. Rappelons que le clonage de singes, à partir de cellules embryonnaires, avait été annoncé dès 1997 [7], mais jamais confirmé ni reproduit, et que divers commentaires faisaient état de graves anomalies chromosomiques apparaissant dès les premiers stades du développement embryonnaire. Dans cet article, les auteurs montraient que le fuseau mitotique était très anormal dans les cellules des embryons obtenus par transfert nucléaire, et que deux protéines impliquées dans ce fuseau et son fonctionnement, NuMA et HSET, y étaient indétectables alors qu'elles sont présentes dans le noyau des embryons normaux. La conclusion était donc que l'énucléation emportait ces protéines indispensables et aboutissait aux anomalies chromosomiques constatées, puis à un arrêt précoce du développement. Chez les autres mammifères, ces protéines sont plus largement réparties dans l'ovocyte et restent donc en quantité suffisante après énucléation. On pouvait donc considérer que le clonage de primates 
(et donc d'humains) était beaucoup plus difficile que celui de vaches, de souris ou de brebis, et que son éventualité se trouvait reportée à un avenir (confortablement) éloigné. Ce résultat achevait par ailleurs de décrédibiliser l'annonce des Raéliens (décembre 2002) prétendant avoir abouti à la naissance d'un enfant cloné - affirmation pour laquelle aucun

$(\rightarrow) \mathrm{m} / \mathrm{s}$ $2003, n^{\circ} 2$, p. 243 élément de preuve n'a jamais été apporté $(\rightarrow)$. L’heure était à la morosité: José Cibelli, l'un des pionniers du clonage thérapeutique à Advanced Cell Genetics, avait quitté cette firme pour l'université du Michigan, tandis que PPL therapeutics, entreprise pionnière du clonage animal avec Megan, Morag, Dolly et Polly ${ }^{3}$, en état de quasi-faillite, vendait l'ensemble de sa propriété industrielle sur le clonage pour moins d'un million de livres anglaises, une misère...

\section{Cette fois, c'est sérieux}

L'article rapportant le travail des laboratoires sud-coréens [1] est, lui, de nature à redonner espoir aux cloneurs, et s'avère autrement probant que ceux d'Advanced Cell Genetics ou de Panos Zavos. Publié par la prestigieuse revue Science, il est très documenté et pour tout dire passablement convaincant. En résumé: les auteurs ont traité au total 242 ovocytes humains, en y injectant une cellule cumulaire autologue; presque tous (213) se sont divisés au moins une fois, et 30 d'entre eux ont atteint le stade blastocyste. À partir de ces derniers, les cellules de la masse interne ont pu être isolées dans 20 cas; finalement, une lignée de cellules souches a été obtenue. On peut noter le grand nombre d'ovocytes employés, fournis par 16 volontaires non rétribuées: les scientifiques d'Advanced Cell Genetics n'avaient pu disposer que d'une vingtaine d'ovules ${ }^{4}$. Cela a permis de tester au total douze protocoles différents, en variant les conditions de culture et d'activation des embryons. L'énucléation, étape très critique si l'on en croit les résultats obtenus chez le singe Rhésus, est effectuée de manière originale: au lieu d'aspirer le noyau de l'ovocyte dans une micropipette, les chercheurs ont pratiqué un petit trou dans sa paroi puis expulsé le noyau par une légère pression sur l'ovocyte. On peut supposer que cette «énucléation douce» laisse dans l'ovocyte suffisamment de NuMA et de HSET pour assurer le bon fonctionnement de l'appareil mitotique lors des divisions ultérieures. Notons aussi l'emploi pour chaque injection d'une cellule cumulaire provenant de la même donneuse. Ces cellules somatiques, qui adhèrent à l'ovocyte et peuvent donc être obtenues lors de son prélèvement, sont celles qui, chez l'animal, donnent le meilleur taux de succès. C'est peut-être lié au fait qu'elles sont naturellement arrêtées à la phase G0, ce qui, d'après Wilmut et Campbell, est particulièrement propice à la reprogrammation du noyau après injection [8]. Le fait que la cellule injectée provienne de la donneuse d'ovocyte peut éviter d'éventuelles

\footnotetext{
${ }^{3}$ Megan et Morag étaient les premiers agneaux clonés, en 1995, à partir de cellules embryonnaires amenées en phase GO. Polly, qui suivit de peu Dolly, était la première brebis clonée et transgénique, produisant dans son lait le facteur IX de la coagulation.

${ }^{4}$ Et avaient payé 4000 dollars US à chaque donneuse..
}

incompatibilités - tout en limitant le champ d'application du procédé au sexe féminin. Enfin, l'intervalle optimal entre injection et activation semble être de deux heures, et cette dernière est réalisée par voie chimique et non par un choc électrique.

\section{Des contrôles convaincants}

Quelques questions restent posées: les embryons obtenus résultentils effectivement du transfert nucléaire, ne s'agirait-il pas simplement d'une parthénogenèse, dont on sait qu'elle peut être déclenchée par les activateurs chimiques utilisés et qu'elle autorise un début de développement de l'embryon? Comme l'ovule et la cellule injectée (donc leurs ADN respectifs) proviennent de la même personne, la confusion serait en principe possible. Mais les réponses apportées sont assez satisfaisantes. Les auteurs ont systématiquement contrôlé l'expulsion du noyau de l'ovocyte, qui est bien énucléé et donc incapable de développement autonome. De plus, les analyses pratiquées sur l'ADN de la lignée de cellules souches obtenue à partir d'un de ces embryons montrent qu'à différents locus hypervariables son ADN est hétérozygote (comme celui de la cellule injectée) alors qu'un embryon résultant d'une parthénogenèse (diploïdisation du jeu haploïde de l'ovule) serait forcément homozygote. L'examen des profils d'expression de plusieurs gènes soumis à l'empreinte parentale va dans le même sens. Il est donc quasiment certain que les embryons obtenus sont bien des clones. Jusqu'à quel point sont-ils normaux? Le fait qu'ils arrivent jusqu'au stade blastocyste (une centaine de cellules) est rassurant, sans bien sûr constituer une preuve absolue: une telle évolution ne serait pas possible s'ils présentaient les aberrations chromosomiques majeures constatées dans les études sur le singe Rhésus.

La mise en culture des cellules de la masse interne des blastocystes, réalisée dans vingt cas, n'a pourtant donné naissance qu'à une seule lignée de cellules souches. Ce taux de succès est très faible puisque, pour les lignées établies à partir de «vrais» embryons (c'est-à-dire après fécondation in vitro), il oscille entre 20 et $50 \%$. Il est fort possible qu'ici encore la reprogrammation joue des tours aux apprentis-cloneurs, que la plupart des embryons obtenus par transfert nucléaire aient suffisamment réorganisé le profil d'expression de la cellule cumulaire pour arriver au stade blastocyste - mais pas assez pour produire de véritables cellules souches. La lignée obtenue présente néanmoins toutes les caractéristiques attendues: caryotype normal, marqueurs de surface caractéristiques et capacité de se différencier en différents tissus (épithélium, muscle, os, cartilage...). Le résultat obtenu est donc scientifiquement très important, même si sa portée pratique reste pour l'instant limitée.

\section{Où en est-on vraiment?}

Le schéma du clonage thérapeutique humain devient donc nettement plus réaliste. Rappelons tout de même qu'il ne s'agit encore que d'un schéma, et non d'une approche thérapeutique utilisable à court terme: l'obtention de cellules souches à partir d'un embryon 
cloné ne représente qu'une étape d'un ensemble complexe, il reste encore à maîtriser en pratique leur différenciation vers le type cellulaire requis pour la thérapie. En tout état de cause, même si la lignée obtenue tient toutes ses promesses, on se voit mal utiliser 242 ovocytes pour obtenir les cellules permettant - peut-être - de traiter un seul malade... II n'empêche, l'impact psychologique de cette réussite est important, on en voit déjà les effets. Du côté des industriels et des chercheurs engagés dans ces travaux, on reprend courage; quant aux discussions sur l'interdiction, ou l'autorisation sous conditions, de la recherche sur le clonage thérapeutique, elles repartent de plus belle. Rappelons que la question n'est toujours pas tranchée sur le plan législatif en France, puisque la révision de la loi de bioéthique de 1995 attend toujours de repasser en deuxième lecture à l'Assemblée Nationale $(\rightarrow)$. Aux États-Unis, comme au niveau de l'ONU, les désaccords sur le clonage thérapeutique ont jus- $(\rightarrow) \mathrm{m} / \mathrm{s}$ 2004, no 3 , p. 374 qu'ici empêché l'adoption de toute législation, y compris celle qui interdirait le clonage reproductif. Huit ans après la naissance de Dolly, le bilan n'est pas fameux, d'autant plus que les résultats coréens réactivent la perspective du clonage reproductif humain en montrant qu'au moins sa première étape n'est pas techniquement hors de portée. Ces péripéties montrent en tous cas que le rythme d'évolution d'une technologie est imprévisible, et qu'il est nécessaire de se préoccuper de son encadrement même lorsqu'elle paraît encore balbutiante, afin de ne pas être pris de court par une évolution plus rapide que prévue. $\diamond$ Ups and downs of therapeutic cloning

\section{RÉFÉRENCES}

1. Hwang WS, Ryu YJ, Park JH, et al. Evidence of a pluripotent human embryonic stem cell line derived from a cloned blastocyst. Sciencexpress 12 février 2004,1094515.

2. Simerly C, Dominko T, Navara C, et al. Molecular correlates of primate nuclear transfer failures. Science 2003; 300: 297.

3. Jordan B. Les marchands de clones. Paris: Seuil, 2003.

4. Atlan H. Le «clonage» thérapeutique. Med Sci (Paris) 2002; 18: 635-8.

5. Cibelli JB, Kiessling AA, Cunniff K, et al. Somatic cell nuclear transfer in humans: pronuclear and early embryonic development. J Regener Med 2001; 2: 25-31.

6. Zavos PM. Human reproductive cloning: the time is near. Reprod Biomed Online $2003 ; 6: 397-8$.

7. Meng L, Ely JJ, Stouffer RL, et al. Rhesus monkeys produced by nuclear transfer. Biol Reprod 1997; 57: 454-9.

8. Campbell KHS, McWhir J, Ritchie WA, et al. Sheep cloned by nuclear transfer from a cultured cell line. Nature 1996; $380: 64-6$

\section{TIRÉS À PART}

B. Jordan

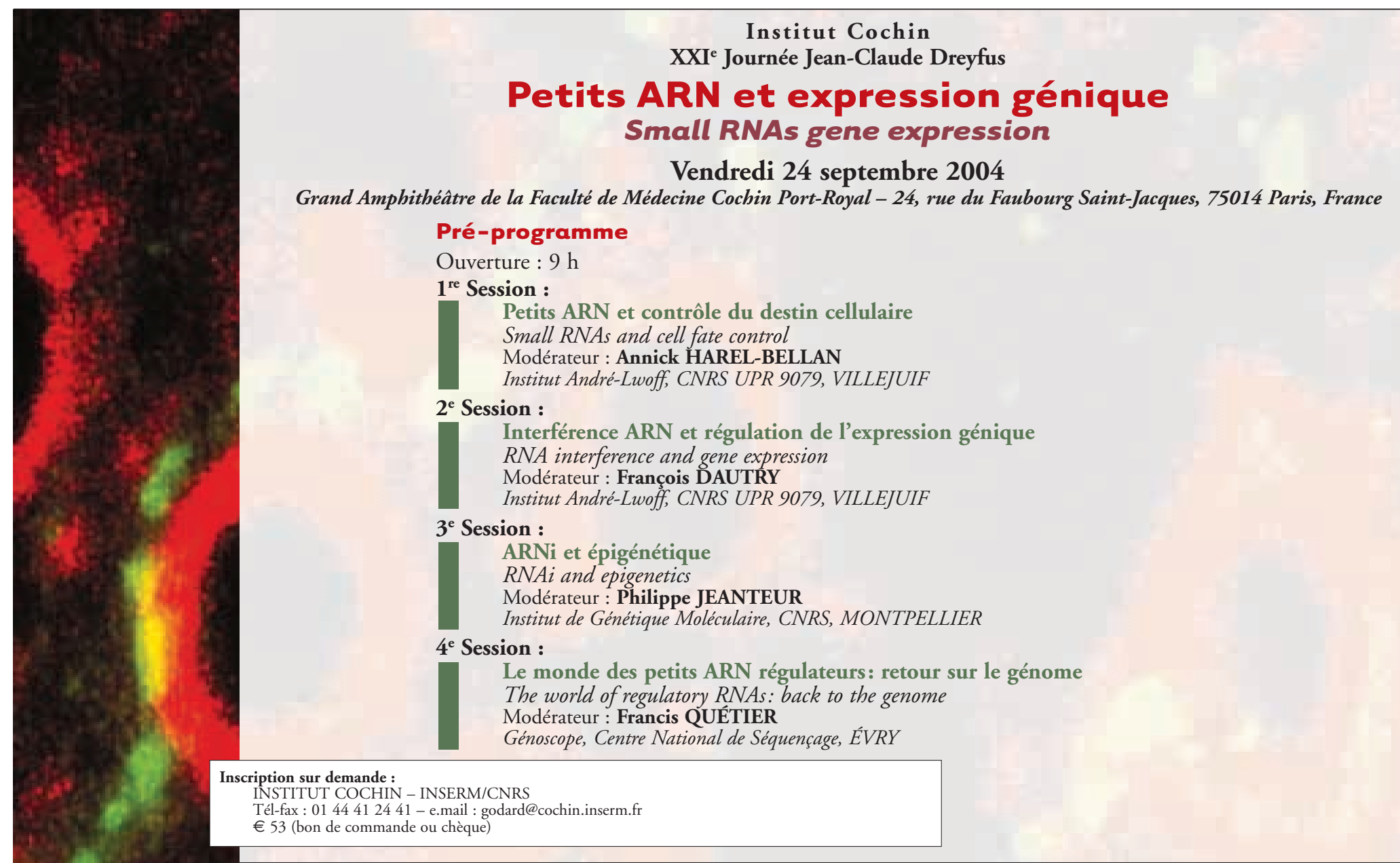

